Consensus control of observer-based mil t i - agent syst em wi th commini cat i on del ay

\begin{tabular}{|l|l|}
\hline 著者 & Naner i kawa Tor u, Yoshi oka Chi ka \\
\hline $\begin{array}{l}\text { j our nal or } \\
\text { publ i cat i on t i t l e }\end{array}$ & Proceedi ngs of the SI CE Annual Conf er ence \\
\hline vol une & 4655069 \\
\hline page $r$ ange & 2414 2416 \\
\hline year & 2008 01- 01 \\
\hline URL & ht t p: //hdl . handl e. net /2297/12649 \\
\hline
\end{tabular}




\title{
Consensus Control of Observer-based Multi-Agent System with Communication Delay
}

\author{
Toru Namerikawa ${ }^{1}$ and Chika Yoshioka ${ }^{1}$ \\ ${ }^{1}$ Division of Electrical Engineering and Computer Science, \\ Graduate School of Natural Science and Technology, \\ Kanazawa University \\ Kakuma-machi, Kanazawa 920-1192 JAPAN. \\ (Tel : +81-76-234-4848; E-mail: toru@t.kanazawa-u.ac.jp)
}

\begin{abstract}
This paper proposes an observer-based consensus control strategy for multi-agent system (MAS) with communication time delay. The condition of stability for MIMO agents is derived by Lyapunov theorem. It gives systematic design procedure under assumed unidirectional network. Furthermore, new consensus control law using observers is proposed for the networked MAS with communication delays.

Experimental results show effectiveness of our proposed output consensus approaches.
\end{abstract}

Keywords: Multi-Agent System, Consensus Control, Output Feedback Communication Delay, Formation Control

\section{INTRODUCTION}

There have been rapidly progresses of new theories that create a fusion of graph theory and system control theory for cooperative control problem of distributed networked control systems[1],[2]. As one of these research works, a multi-agent system (MAS) is one of significant topics which each agent autonomously works by using information of other agents over the network [3]-[9].

Let consider an agent as a vehicle in MAS, then it is possible to apply formation control problem [3]-[4]. Formation control problems expect at various fields, e.g. satellites, airship, intelligent transport systems and load carriage. Therefore control problem of MAS is useful and practically problem.

In this paper, we proposes an observer-based consensus control strategy for multi-agent system with communication time delay. In the networked MAS, consensus means to reach an agreement regarding a certain quantity of interest that depends on the state of of dynamical agents. Consensus algorithm with graph theory has been studied as a control problem of MAS in [5]-[8]. In [5] and [6], proposes a new control law to which consensus algorithm [7] is enhanced. It was shown in [5] that stability of MAS could be checked graphically using Nyquist-like criterion. Depending on the agent system, it is difficult to design controllers using Nyquist-like criterion. Also, [6] derive condition of stability of MAS using Lyapunov stability theorem under assumed unidirectional network (balanced graph) where an agent is second-order system. So the MAS is much less common than [5]. Therefore we consider that each agent is MIMO linear system and propose the condition of stability using Lyapunov stability theorem. Using Lyapunov theorem, controller design is easier than [5] under assumed unidirectional network.

In addition, there are some problem in network structure of MAS: communication delay and constrained communication. In this paper, new consensus control law us- ing observers is proposed for constrained communication where communication delays are occurred in inter-agent communications. In [9], a Lyapunov-like criterion was derived for stability conditon in MAS with constant communication delay. The paper[4] considers parallel estimators for formation control of MAS where the agent is spacecraft. A spacecraft estimate states of other space that connected network. Hence order of the estimator under assumed large network is larger than other estimator under assumed small network. Therefore, the proposed observer-based output control strategy is much simpler than the methos in [4]. Finally, the experimental results show effectiveness of our proposed control law.

\section{GRAPH THEORY}

Graph is useful to represent network structures [11]. Let $G=(\mathscr{V}, \mathscr{A})$ be a graph of order $N$ with the set of nodes $\mathscr{V}=\left\{v_{1}, \cdots, v_{N}\right\}$ and set of $\operatorname{arcs} \mathscr{A} \subseteq \mathscr{V} \times \mathscr{V}$. An $\operatorname{arc}\left(v_{i}, v_{j}\right)$ of $G$ is shown by an arrow drawn from node $v_{j}$ to node $v_{i}$. The set of neighbors of node $v_{i}$ is denoted by $\mathscr{N}_{i}=\left\{v_{j} \in \mathscr{V}:\left(v_{j}, v_{i}\right) \in \mathscr{A}\right\}$.

In MAS, node $v_{i}$ means $i$ th agent and arc $\left(v_{i}, v_{j}\right)$ is communication that $j$ th agent send some information to $i$ th agent.

In this paper, we use Graph Laplacian for graph structure expressed mathematically. Graph Laplacian $L=\left[l_{i j}\right]$ is matrix that $l_{i i}=\sum_{j \neq i} a_{i j}, l_{i j}=-a_{i j}(i \neq j)$ where $a_{i j}=1\left(v_{j} \in \mathscr{N}_{i}\right)$ and $a_{i j}=0\left(v_{j} \notin \mathscr{N}_{i}\right)$. If $L=L^{T}$, Graph $G$ is called Undirected graph, otherwise $G$ is directed graph (Digraph). The undirected graph called connected graph if it is possible to reach any agent starting from any other agent by traversing network. In digraph case, the directed graph called strongly connected graph.

Assuming that the graph is (strongly) connected graph, Graph Laplacian $L$ satisfies the following properties:

i) There is unique zero-eigenvalue of $L$

ii) All eigenvalues of $L$ are nonnegative. 
iii) An eigenvector of zero-eigenvalue of $L$ is $N$ th column vector of all ones.

In this paper, we express eigenvalues of $N \times N$ symmetric matrix $a$ as

$\lambda_{1}(a) \leq \lambda_{2}(a) \leq \cdots \leq \lambda_{N}(a)$.

where $\lambda_{k}(a)$ is $k$ th eigenvalue of $a$. It is well-known that eigenvalues of Graph Laplacian $L$ in connected graph can be presented as

$0=\lambda_{1}(L)<\lambda_{2}(L) \leq \cdots \leq \lambda_{N}(L)$.

\section{MULTI-AGENT SYSTEM}

In this paper, the Networked MAS is treated. A plant is the MAS which consists $N$ agents under following assumption.

[Assumption 1] The network structure of MAS is (strongly) connected graph.

Consider $i$ th agent system as

$\dot{x}_{i}=A x_{i}+B u_{i}$,

where $A \in \mathbb{R}^{n \times n}, B \in \mathbb{R}^{n \times m} . x_{i} \in \mathbb{R}^{n}, u_{i} \in \mathbb{R}^{m}$ are $i$ th state and input. Let $x=\left[x_{1}^{T} \cdots x_{N}^{T}\right]^{T}$ and $u=\left[\begin{array}{lll}u_{1}^{T} & \cdots & u_{N}^{T}\end{array}\right]^{T}$, then MAS can be presented as

$\dot{x}=\left(I_{N} \otimes A\right) x+\left(I_{N} \otimes B\right) u$,

where $I_{N}$ is $N$ th-order unit matrix and $\otimes$ is Kronecker product.

Throughout this paper, we assume that the agent (3) satisfies the following properties:

\section{[Assumptions 2]}

i) There is no positive real eigenvalue of $A$.

ii) $(A, B)$ is controllable.

In this paper, stability of MAS means

$\lim _{t \rightarrow \infty} x=\mathbf{1} \otimes \alpha$

where $\mathbf{1}$ is $N$ th column vector of all ones and $\alpha \in \mathbb{R}^{n}$ is any vector and we define that $\alpha$ is consensus vector. We called consensus of MAS if system (4) achieve Eq. (5).

\section{STABILITY OF MULTI-AGENT SYSTEM}

First, we utilize a consensus algorithm[7] as

$u_{i}=-K \sum_{j \in \mathscr{N}_{i}}^{N}\left(x_{i}-x_{j}\right)$,

where $K \in \mathbb{R}^{m \times n}$ is the controller gain. Consensus algorithm (6) can be written in matrix form as

$u=-(L \otimes K) x$.

By Eq. (7), MAS (4) is expressed as

$\dot{x}=\left(\left(I_{N} \otimes A\right)-(L \otimes B K)\right) x$.

Following theorem is derived about consensus of MAS (8).
[Theorem 1] Assume that $\Lambda_{e}+\Lambda_{e}^{T}>0$ and MAS (8) satisfy [Assumption 1-2]. Then MAS (8) achieve consensus

$\alpha=N^{1 / 2} \exp (A t)\left(l_{1}^{T} \otimes I_{n}\right) x(0)$

if there is positive definite matrix $P$ such that

$A^{T} P+P A-\lambda_{1}\left(\Lambda_{e} \Lambda_{e}^{T}\right) P B B^{T} P<0$

and controller gain $K$ is

i) Digraph case

$K=\frac{\lambda_{N-1}\left(\Lambda_{e} \Lambda_{e}^{T}\right)}{\lambda_{1}\left(\Lambda_{e}+\Lambda_{e}^{T}\right)} B^{T} P$

ii) Undirected graph case

$K=\frac{1}{2} \lambda_{N}(L) B^{T} P$,

where $\Lambda_{e} \in \mathbb{R}^{N-1 \times N-1}$ is matrix that is gotten by Jordan form $\Lambda=\left[\begin{array}{cc}0 & 0 \\ 0 & \Lambda_{e}\end{array}\right]=S L S^{-1}$ of graph Laplacian $L$, where $S$ is any regular matrix which first row vector is $N^{-1 / 2} \mathbf{1} . \quad l_{1}$ is left-eigenvector corresponding to zero-eigenvalue of $L$ where $l_{1}^{T} N^{-1 / 2} \mathbf{1}=1$.

proof: Jordan form $\Lambda$ of graph Laplacian $L$ is expressed as

$\Lambda=\left[\begin{array}{cc}0 & 0 \\ 0 & \Lambda_{e}\end{array}\right]=S^{-1} L S$

Now, we consider coordinate transformation $z=$ $\left(S^{-1} \otimes I_{n}\right) x$, then the system (8) is expressed as:

$$
\begin{aligned}
\dot{z} & =\left(\left(S^{-1} S \otimes A\right)-\left(S^{-1} L S \otimes B K\right)\right) z \\
& =\left(\left(I_{N} \otimes A\right)-(\Lambda \otimes B K)\right) z,
\end{aligned}
$$

where $z=\left[\begin{array}{ll}z_{1}^{T} & z_{e}^{T}\end{array}\right]^{T} \in \mathbb{R}^{N n}, z_{e}=\left[\begin{array}{lll}z_{2}^{T} & \cdots & z_{N}^{T}\end{array}\right]^{T} \in \mathbb{R}^{(N-1) n}$. Eq. (14) can be divided into two equations:

$$
\begin{aligned}
& \dot{z}_{1}=A z_{1} \\
& \dot{z}_{e}=\left(\left(I_{N-1} \otimes A\right)-\left(\Lambda_{e} \otimes B K\right)\right) z_{e},
\end{aligned}
$$

If $z_{e}$ converge to equilibrium $\left(z_{e} \rightarrow 0\right.$ as $\left.t \rightarrow \infty\right)$, MAS (8) achieves consensus by Eqs. (15)-(16) and Assumption 2-i). Therefore, we get

$$
\begin{aligned}
\lim _{t \rightarrow \infty} x & =\lim _{t \rightarrow \infty}\left(S \otimes I_{n}\right) z=\left(N^{-1 / 2} \mathbf{1} \otimes I_{n}\right) z_{1} \\
& =\mathbf{1} \otimes N^{-1 / 2} e^{A t}\left(l_{1}^{T} \otimes I_{n}\right) x(0)
\end{aligned}
$$

This means each agent goes to a consensus. If we can prove an asymptotical stability of Eq. (16), then MAS achieves consensus. Consider a Lyapunov function $V_{1}$ as:

$V_{1}=z_{e}^{T}\left(I_{N-1} \otimes P\right) z_{e}$.

Differentiate $V_{1}$ along the trajectories, we get

$\dot{V}_{1}=z_{e}^{T}\left(I_{N-1} \otimes\left(A^{T} P+P A\right)-\Lambda_{e}^{T} \otimes K^{T} B^{T} P-\Lambda_{e} \otimes P B K\right) z_{e}$.

If there is positive definite matrix $P$ such that

$I_{N-1} \otimes\left(A^{T} P+P A\right)-\left(\Lambda_{e}^{T} \otimes K^{T} B^{T} P\right)-\left(\Lambda_{e} \otimes P B K\right)<0$.

Then the system (16) is stable. 
Next, we consider stabilization condition of control gain $K$. By assumption 2-ii), $\left(A, \lambda_{1}\left(\Lambda_{e} \Lambda_{e}^{T}\right)^{-1 / 2} B\right)$ is stabilizable. Therefore, there is positive definite matrix $P$ such that

$A^{T} P+P A-\lambda_{1}\left(\Lambda_{e} \Lambda_{e}^{T}\right) P B B^{T} P<0$.

Because of $\Lambda_{e} \Lambda_{e}^{T}>0$, Eq. (21) is expressed as:

$$
\begin{aligned}
0 & >\left(I_{N-1} \otimes A^{T} P+P A\right)-\left(\lambda_{1}\left(\Lambda_{e} \Lambda_{e}^{T}\right) \otimes P B B^{T} P\right) \\
& \geq\left(I_{N-1} \otimes A^{T} P+P A\right)-\left(\Lambda_{e} \Lambda_{e}^{T} \otimes P B B^{T} P\right) .
\end{aligned}
$$

First, let consider gain $K$ in digraph case.

\section{i) Digraph}

(22) can be represent as

$$
\begin{aligned}
0 & >\left(I_{N-1} \otimes A^{T} P+P A\right)-\left(\frac{\Lambda_{e} \Lambda_{e}^{T}}{\lambda_{1}\left(\Lambda_{e}+\Lambda_{e}^{T}\right)} \lambda_{1}\left(\Lambda_{e}+\Lambda_{e}^{T}\right) \otimes P B B^{T} P\right) \\
& \geq\left(I_{N-1} \otimes A^{T} P+P A\right)-\left(\frac{\Lambda_{e} \Lambda_{e}^{T}}{\lambda_{1}\left(\Lambda_{e}+\Lambda_{e}^{T}\right)}\left(\Lambda_{e}+\Lambda_{e}^{T}\right) \otimes P B B^{T} P\right) \\
& \geq\left(I_{N-1} \otimes A^{T} P+P A\right)-\left(\Lambda_{e}+\Lambda_{e}^{T}\right) \otimes\left(\frac{\lambda_{N-1}\left(\Lambda_{e} \Lambda_{e}^{T}\right)}{\lambda_{1}\left(\Lambda_{e}+\Lambda_{e}^{T}\right)} P B B^{T} P\right)
\end{aligned}
$$

If there is positive definite matrix $P$ such that Eq. (21) and controller gain $K$ define Eq. (11), MAS (8) satisfy Eq. (20). Therefore MAS (8) satisfy consensus.

It is clear that $\Lambda_{e}+\Lambda_{e}^{T}$ is positive definite matrix if there is no duplicate eigenvalue of Graph Laplacian $L$. And also $\Lambda_{e}+\Lambda_{e}^{T}$ is positive definite matrix if Graph is balanced graph by the property of Graph Laplacian. Therefore, there is $\Lambda_{e}+\Lambda_{e}^{T}>0$ at least.

Next, we define controller gain $K$ in undirected graph.

ii) Undirected graph

Graph Laplacian $L$ of undirected graph is symmetric matrix. Therefore, $\Lambda_{e}>0$ is diagonal matrix. Hence Eq. (22) can be written as

$$
\begin{aligned}
0 & >\left(I_{N-1} \otimes A^{T} P+P A\right)-\left(\Lambda_{e}^{2} \otimes P B B^{T} P\right) \\
& \geq\left(I_{N-1} \otimes A^{T} P+P A\right)-\left(\Lambda_{e} \otimes \lambda_{N-1}\left(\Lambda_{e}\right) P B B^{T} P\right) .
\end{aligned}
$$

By Eq. (24), if there is positive definite matrix $P$ such that Eq. (21) and control gain $K$ define Eq. (12) when MAS (8) satisfies Eq. (20). Therefore MAS (8) satisfies a consensus.

\section{STABILITY OF MULTI-AGENT SYSTEM WITH COMMUNICATION DELAY}

Consider the stability analysis of MAS with communication delay. Here a communication time delay is treated to be constant and a control law is proposed by using maximum tolerable communication delay $\tau$.

The proposed control law of $i$ th agent with communication delay can be represented as

$u_{i}(t)=-K \sum_{j \in \mathscr{N}_{i}}^{N}\left(x_{i}(t-\tau)-x_{j}(t-\tau)\right)$.

Hence, the control law of MAS is expressed

$u(t)=-(L \otimes K) x(t-\tau)$.

Therefore, MAS with communication delay can be represented as

$\dot{x}(t)=\left(I_{N} \otimes A\right) x(t)-(L \otimes B K) x(t-\tau)$.
We analysis that MAS (27) achieve consensus.

[Theorem 2] Assume that MAS (27) satisfy [Assumptions 1-2]. Then MAS (27) achieve consensus if $A-\lambda_{1}\left(\Lambda_{e} \Lambda_{e}^{T}\right)^{1 / 2} B K$ is stable and there is positive definite matrix $P$ such that

$$
\begin{aligned}
& {\left[\begin{array}{cc}
\Phi & P B K \\
K^{T} B^{T} P & -\frac{r_{1} r_{2}}{\left(r_{1}+r_{2}\right) \tau \lambda_{N-1}\left(\Lambda_{e} \Lambda_{e}^{T}\right)} I_{n}
\end{array}\right]<0} \\
& \Phi=A^{T} P+P A-\lambda_{1}\left(\Lambda_{e} \Lambda_{e}^{T}\right)^{1 / 2}\left(K^{T} B^{T} P+P B K\right) \\
& +r_{1} \tau A^{T} A^{T}+r_{2} \tau \lambda_{N-1}\left(\Lambda_{e}^{T} \Lambda_{e}\right) K^{T} B^{T} B K
\end{aligned}
$$

where $r_{1}, r_{2}$ are positive scalar.

Proof: MAS (27) can be divided into two equation well as theorem 1 by coordinate transformation $z=\left(S^{-1} \otimes I_{n}\right) x$.

$$
\begin{aligned}
& \dot{z}_{1}(t)=A z_{1}(t) \\
& \dot{z}_{e}(t)=\left(I_{N-1} \otimes A\right) z_{e}(t)-\left(\Lambda_{e} \otimes B K\right) z_{e}(t-\tau)
\end{aligned}
$$

Therefore it is clear that stability of Eq. (30) and MAS (27) achieve consensus are equivalent. Consequently, we prove stability of Eq. (30). Let

$$
\begin{aligned}
& z_{e}(t-\tau)=z_{e}(t) \\
& \quad-\int_{t-\tau}^{t}\left(I_{N-1} \otimes A\right) z_{e}(\theta)-\left(\Lambda_{e} \otimes B K\right) z_{e}(\theta-\tau) d \theta .
\end{aligned}
$$

Then, Eq. (30) can be expressed as

$$
\begin{aligned}
& \dot{z}_{e}(t)=\left(\left(I_{N-1} \otimes A\right)-\left(\Lambda_{e} \otimes B K\right)\right) z_{e}(t) \\
& +\left(\Lambda_{e} \otimes B K\right) \int_{t-\tau}^{t}\left(I_{N-1} \otimes A\right) z_{e}(\theta)-\left(\Lambda_{e} \otimes B K\right) z_{e}(\theta-\tau) d \theta .
\end{aligned}
$$

Let us define a candidate of Lyapunov-like function as

$$
\begin{aligned}
V_{2}\left(z_{e}, t\right) & =z_{e}^{T}(t)\left(I_{N-1} \otimes P\right) z_{e} x(t) \\
& +r_{1} \int_{t-\tau}^{t} \int_{\theta}^{t}\left\|\left(I_{N-1} \otimes A\right) z_{e}(s)\right\|^{2} d s d \theta \\
& +r_{2} \int_{t-\tau}^{t} \int_{\theta}^{t}\left\|\left(\Lambda_{e} \otimes B K\right) z_{e}(s)\right\|^{2} d s d \theta
\end{aligned}
$$

where $P$ is positive definite matrix. Then, the derivative $V_{2}$ along the trajectories as

$$
\begin{aligned}
& \dot{V}_{2}\left(z_{e}, t\right)=z_{e}^{T}\left(I_{N-1} \otimes\left(A^{T} P+P A\right)\right) z_{e} \\
& -z_{e}^{T}\left(\Lambda_{e}^{T} \otimes K^{T} B^{T} P\right) z_{e}+z_{e}^{T}\left(\Lambda_{e} \otimes P B K\right) z_{e} \\
& +2 z_{e}^{T}\left(I_{N-1} \otimes P\right)\left(\Lambda_{e} \otimes B K\right) \\
& \quad \times \int_{t-\tau}^{t}\left(I_{N-1} \otimes A\right) z_{e}(\theta)-\left(\Lambda_{e} \otimes B K\right) z_{e}(t-\tau) d \theta \\
& +r_{1} \tau\left\|\left(I_{N-1} \otimes A\right) z_{e}(t)\right\|^{2}+r_{2} \tau \|\left(\left(\Lambda_{e} \otimes B K\right) z_{e}(\theta) \|^{2} .\right.
\end{aligned}
$$

By Lyapunov krasovskii theorem, if there is positive definite matrix $P$ such tha

$$
\begin{aligned}
& \left(I_{N-1} \otimes\left(A^{T} P+P A\right)\right)-\Lambda_{e}^{T} \otimes K^{T} B^{T} P-\Lambda_{e} \otimes P B K \\
& +\tau\left(\frac{1}{r_{1}}+\frac{1}{r_{2}}\right)\left(I_{N-1} \otimes P\right)\left(\Lambda_{e} \otimes B K\right)\left(\Lambda_{e}^{T} \otimes K^{T} B^{T}\right)\left(I_{N-1} \otimes P\right) \\
& +r_{1} \tau\left(I_{N-1} \otimes A^{T} A\right)+r_{2} \tau\left(\Lambda_{e}^{T} \Lambda_{e} \otimes K^{T} B^{T} B K\right)<0,
\end{aligned}
$$

then MAS (27) satisfy consensus.

Next, assumed $A-\lambda_{1}\left(\Lambda_{e} \Lambda_{e}^{T}\right)^{1 / 2}$ is stable. Then the following inequality is derived by (35).

$$
\begin{aligned}
& A^{T} P+P A-\lambda_{1}\left(\Lambda_{e} \Lambda_{e}^{T}\right)^{1 / 2}\left(K^{T} B^{T} P+P B K\right) \\
& +\tau\left(\frac{1}{r_{1}}+\frac{1}{r_{2}}\right) \lambda_{N-1}\left(\Lambda_{e} \Lambda_{e}^{T}\right) P B K K^{T} B^{T} P \\
& +r_{1} \tau A^{T} A+r_{2} \tau \lambda_{N-1}\left(\Lambda_{e}^{T} \Lambda_{e}\right) K^{T} B^{T} B K<0
\end{aligned}
$$


It is clear that (36) is satisfied then (35) is also satisfied. Hence, using schur complement, Theorem 2 is derived by (36).

(36) is more conservative than (35). However, computational load of (36) according to increase of agents is lower than (35).

\section{OBSERVER-BASED MULTI-AGENT SYSTEM}

Let consider output feedback of consensus problem for reducing of communication traffic. Two control law in Section II and III are state feedback of consensus problem. In these case, an agent sends state and receives other agent state. Assumed MAS which consists $N$ agents where an agent has $n$ states and the network structure is complete graph, then an agent gets $n(N-1)$ information and send $n(N-1)$ information. If an agent sends $m$ outputs where $m<n$ case, then an agent get $m(N-1)$ information and send $m(N-1)$ information. Hence using output information of agents, the communication traffic is reduced. Therefore, let consider $i$ th information of output $y_{i}$ and $i$ th controlled information $Z_{i}$ as

$y_{i}=C x_{i}, \quad Z_{i}=\sum_{j \in \mathscr{N}_{i}}^{N}\left(y_{i}-y_{j}\right)=\sum_{j \in \mathscr{N}_{i}}^{N} C\left(x_{i}-x_{j}\right)$.

where $C \in \mathbb{R}^{m \times n}$. Then, the MAS can be expressed as

$\dot{x}=\left(I_{N} \otimes A\right) x+\left(I_{N} \otimes B\right) u$

$y=\left(I_{N} \otimes C\right) x$

$Z=\left(L \otimes I_{n}\right) y=(L \otimes C) x$,

where $y=\left[\begin{array}{llll}y_{1}^{T} & y_{2}^{T} & \cdots & y_{N}^{T}\end{array}\right]^{T}, Z=\left[\begin{array}{lll}Z_{1}^{T} & Z_{2}^{T} \cdots Z_{N}^{T}\end{array}\right]^{T}$ and this MAS satisfy under following assumption.

[Assumption 3] $(A, C)$ is observable.

$i$ th observer-based control law is proposed as

$$
\begin{aligned}
& \dot{\hat{x}}_{F i}=(A-H C) \hat{x}_{F i}+H \sum_{j \in \mathscr{N}_{i}}^{N}\left(y_{i}-y_{j}\right)+B u_{i} \\
& u_{i}=-K \hat{x}_{F i},
\end{aligned}
$$

where $\hat{x}_{F i}$ is estimation vector that estimate $\sum_{j \in \mathscr{N}_{i}}^{N}\left(x_{i}-\right.$ $\left.x_{j}\right)$ and $H \in \mathbb{R}^{n \times m}$ is observer gain. Then, the observerbased control law of MAS can be expressed as

$$
\begin{aligned}
& \dot{\hat{x}}_{F}=\left(I_{N} \otimes A-H C\right) \hat{x}_{F}+(L \otimes H C) x+\left(I_{N} \otimes B\right) u \\
& u=-\left(I_{N} \otimes K\right) \hat{x}_{F},
\end{aligned}
$$

where $\hat{x}_{F}=\left[\begin{array}{llll}x_{F 1}^{T} & x_{F 2}^{T} & \cdots & x_{F N}^{T}\end{array}\right]^{T}$ is estimation vector that estimate $\left(L \otimes I_{n}\right) x$. Therefore, observer-based MAS is represented as

$\left[\begin{array}{c}\dot{x} \\ \hat{\hat{x}}_{F}\end{array}\right]=\left[\begin{array}{cc}I_{N} \otimes A & I_{N} \otimes B K \\ L \otimes H C & I_{N} \otimes(A-H C-B K)\end{array}\right]\left[\begin{array}{c}x \\ \hat{x}_{F}\end{array}\right]$.

Let us consider stability of MAS (41).

[Theorem 3] MAS (38) satisfy [Assumption 13]. Then MAS (41) achieve consensus if $A-$ $\lambda_{1}\left(\Lambda_{e} \Lambda_{e}^{T}\right)^{1 / 2} B K$ and $A-H C$ are stability.
Proof: Let $x_{F}=\left(L \otimes I_{n}\right) x$ and $u_{F}=\left(L \otimes I_{m}\right) u$, then the MAS (38) can be expressed as

$\dot{x}_{F}=\left(I_{N} \otimes A\right) x_{F}+\left(I_{N} \otimes B\right) u_{F}$

$Z=\left(I_{N} \otimes C\right) x_{F}$.

The system (42) can be designed observers. Assumed the MAS (38) satisfy that $A-H C$ is stable. Hence, $\hat{x}_{F}$ achieve

$\lim _{t \rightarrow \infty} \hat{x}_{F}=x_{F}=\left(L \otimes I_{n}\right) x$.

Therefore, it is clear that MAS (41) achieves consensus by theorem 1 .

[Remark 1] It is defined that $x_{F}=\left(L \otimes I_{n}\right) x(t-\tau)$. Then a MAS with communication delay satisfy consensus. Then the control law of observer based MVS can be expressed as

$$
\begin{aligned}
& \dot{\hat{x}}_{F}=\left(I_{N} \otimes A-H C\right) \hat{x}_{F}+(L \otimes H C) x(t-\tau)+\left(I_{N} \otimes B\right) u \\
& u=-\left(I_{N} \otimes K\right) \hat{x}_{F} .
\end{aligned}
$$

Then, observer-based MAS with communication delay can be represented as

$$
\begin{aligned}
& {\left[\begin{array}{c}
\dot{x}(t-\tau) \\
\dot{\hat{x}}_{F}
\end{array}\right]=\left[\begin{array}{cc}
I_{N} \otimes A & I_{N} \otimes B K \\
L \otimes H C & I_{N} \otimes(A-H C-B K)
\end{array}\right]} \\
& \times\left[\begin{array}{c}
x(t-\tau) \\
\hat{x}_{F}
\end{array}\right] .
\end{aligned}
$$

[Theorem 4] MAS (38) satisfy [Assumption 13]. Then MAS (45) achieve consensus if $A-$ $\lambda_{1}\left(\Lambda_{e} \Lambda_{e}^{T}\right)^{1 / 2} B K$, there is positive definite matrix $P$ such that (28) and $A-H C$ are stability.

Proof: It is obvious that MAS (45) achieves consensus by Theorem 2 and 3 .

\section{EXAMPLE AND ITS EXPERIMENTS}

\subsection{Multi-vehicle system}

We consider a four-vehicle formation problem and a two-wheel vehicle is treated as an agent as in Fig. 1 (left below).

It is well-known that two-wheel vehicle has nonholonomic property. Assume that $N$ vehicles can be represented same dynamics and there are no friction, then $i$ th vehicle model can be expressed as

$$
\left[\begin{array}{c}
\dot{x}_{r i} \\
\dot{y}_{r i} \\
\dot{\theta}_{r i}
\end{array}\right]=\left[\begin{array}{cc}
\cos \theta_{r i} & 0 \\
\sin \theta_{r i} & 0 \\
0 & 1
\end{array}\right]\left[\begin{array}{c}
v_{i} \\
\omega_{i}
\end{array}\right],
$$

where $\left(x_{r i}, y_{r i}\right)$ is center of gravity of $i$ th vehicle, $\theta_{r i}$ its orientation, control input are its velocity $v_{i}$ and its angular velocity $\omega_{i}$.

Based on an idea of Virtual Structure, we consider Virtual Vehicle (VV) at each vehicles as Fig. 1 (upper right). If positional relationship between a real vehicle and a VV is given as Fig. 1, then position of center of gravity and angle of $\mathrm{VV}$ are expressed as

$$
\left[\begin{array}{c}
x_{v i} \\
y_{v i} \\
\theta_{v i}
\end{array}\right]=\left[\begin{array}{c}
x_{i}+x_{d i} \cos \theta_{r i}-y_{d i} \sin \theta_{r i} \\
y_{i}+x_{d i} \sin \theta_{r i}+y_{d i} \cos \theta_{r i} \\
\theta_{r i}
\end{array}\right] \text {. }
$$




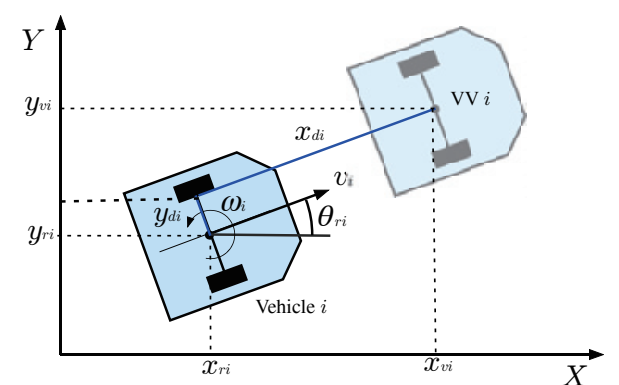

Fig. $1 i$ th Real Vehicle and Virtual Vehicle

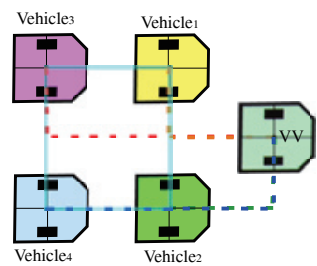

Fig. 2 Target $r_{r i}=0$

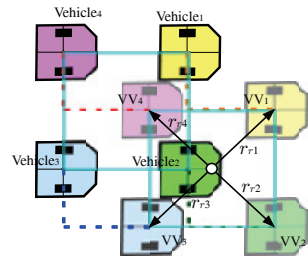

Fig. 3 Target $r_{r i} \neq 0$

Derivatative of (47) gives

$$
\begin{aligned}
{\left[\begin{array}{c}
\dot{x}_{v i} \\
\dot{y}_{v i} \\
\dot{\theta}_{v i}
\end{array}\right] } & =\left[\begin{array}{c}
B_{v i} \\
B_{\theta}
\end{array}\right]\left[\begin{array}{c}
v_{i} \\
\omega_{i}
\end{array}\right] \\
B_{v i} & =\left[\begin{array}{cc}
\cos \theta_{r i} & -x_{d i} \sin \theta_{r i}-y_{d i} \cos \theta_{r i} \\
\sin \theta_{r i} & x_{d i} \cos \theta_{r i}-y_{d i} \sin \theta_{r i}
\end{array}\right] \\
B_{\theta} & =\left[\begin{array}{ll}
0 & 1
\end{array}\right] .
\end{aligned}
$$

If $x_{d i} \neq 0, B_{v i}$ is regular matrix. In this paper, consider formation problem at this VS system (48). Let center of gravity of VV $r_{v i}=\left(x_{v i}, y_{v i}\right)$, VV's velocity $v_{v i}$ and

$\dot{v}_{v i}=u_{v i},\left[v_{i} \omega_{i}\right]^{T}=B_{v i}^{-1} v_{v i}$.

where $u_{v i}$ is new input of VV. Then dynamics of center gravity of $\mathrm{VV}$ can be presented as

$\dot{v}_{v i}=u_{v i}, \dot{r}_{v i}=v_{v i}$.

This dynamics (50) is a linear system.

For VV's uniform motion, we define $u_{v i}$ as

$u_{v i}=-k_{v r}\left(v_{v i}-v^{*}\right)+u_{i}$,

where $k_{v r}>0$ is design parameter, $v^{*} \neq 0$ is reference velocity and $u_{i}$ is new input for consensus. Let $v_{e i}=v_{v i}-$ $v^{*}, \tilde{r}_{v i}=r_{v i}-r_{r i}$, where $r_{r i}$ is reference relative deviation for VV's system achieve any formation. Then the system (50) can be expressed as

$$
\begin{aligned}
& \frac{d}{d t}\left[\begin{array}{l}
\tilde{r}_{v i} \\
v_{e i}
\end{array}\right]=\left[\begin{array}{cc}
0 & 1 \\
-k_{v r} & 0
\end{array}\right] \otimes I_{2}\left[\begin{array}{l}
\hat{r}_{v i} \\
v_{e i}
\end{array}\right]+\left[\begin{array}{l}
0 \\
1
\end{array}\right] \otimes I_{2} u_{i} \\
& y_{i}=\left[\begin{array}{ll}
1 & 0
\end{array}\right] \otimes I_{2}\left[\begin{array}{c}
\tilde{r}_{v i} \\
v_{e i}
\end{array}\right] .
\end{aligned}
$$

If $\mathrm{VVs}$ achieve

$\lim _{t \rightarrow \infty} \tilde{r}_{v i}=\tilde{r}_{v j}, \lim _{t \rightarrow \infty} v_{e i}=v_{e j}$

then real vehicles make formation as Figs.2,3.

Because of the experimental setup can not be detected vehicles's velocities, we use observer based controller for achieve uniform motion of $i$ th vehicle. Let be

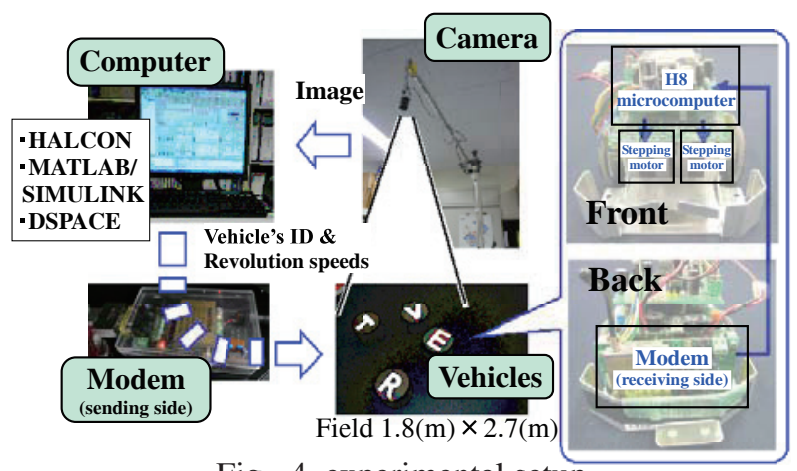

Fig. 4 experimental setup

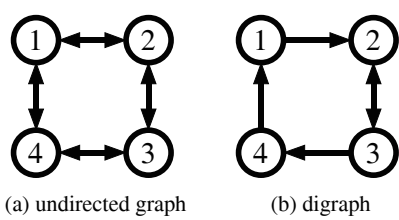

Fig. 5 Network structure

$x_{i}=\left[\begin{array}{lll}\tilde{r}_{v i}^{T} & v_{e i}^{T} & \hat{r}_{v i}^{T} \hat{v}_{e i}^{T}\end{array}\right]^{T}$, then $i$ th vehicle system can be expressed by Eq. (4), where

$$
\begin{aligned}
A & =\left[\begin{array}{cccc}
0 & 1 & 0 & 0 \\
0 & 0 & -k_{v r} & 0 \\
h_{1} & 0 & -h_{1} & 1 \\
h_{2} & 0 & -h_{2}-k_{v r} & 0
\end{array}\right] \otimes I_{2}, B=\left[\begin{array}{l}
0 \\
1 \\
0 \\
1
\end{array}\right] \otimes I_{2}, \\
C & =\left[\begin{array}{llll}
1 & 0 & 0 & 0
\end{array}\right] \otimes I_{2},
\end{aligned}
$$

$\hat{\tilde{r}}_{v i}$ is estimate vector of $\tilde{r}_{v i}, \hat{v}_{e i}$ is estimate vector of $v_{e i}$, $h_{1}, h_{2}>0$ are design parameters. In observer combined system case, $(A, B)$ is not controllable but stabilizable if $\left(A_{l}, B_{l}\right)$ is controllable where $A_{l}, B_{l}$ is system matrix before observer combined system. In this case, assumption 2-ii) can be replaced controllable to stabilizable. And also $(A, C)$ is observable.

\subsection{Experimental Evaluation}

Fig.4 shows our experimental setup. This experiment shows the effectiveness of observer-based MAS with communication delay (Section VI). Network structures are considered two pattern: undirected graph and digraph as shown in Fig. 5. We consider virtual network and communication delay because it can not use intervehicle communication in our experimental setup. And inter-vehicle communication delay $\tau_{i j}$ to $i$ th agent from $j$ th agent is defined as

$\tau_{i j}=0.1\left\|r_{v i}-r_{v j}\right\|+0.1$.

For satisfy this conditions, maximum communication delay is set $\tau=0.3[\mathrm{~s}]$. Let make set to parameters satisfy Theorem 4, these parameters are shown in Table I

Figs. 6-11 show our experimental result. " $\times$ "and "०" are first position and final position of real vehicles in Figs. 6,9 . In undirected graph case, real vehicles achieve formation. It is clear in Figs. 6, 7. In Fig. 7, VV achieve consensus. And also Fig. 8 shows calculated velocities and gives that VVs achieve reference velocity. In digraph case, Fig. 9 shows Trajectories, VV do not completely make formation in the experiment field. Fig. 10 shows 
Table 1 Parameters

\begin{tabular}{|c|c|}
\hline VV's parameter $\left(x_{d i}, y_{d i}\right)$ & $(0.05,0)[\mathrm{m}]$ \\
\hline Reference VV's velocity $v^{*}$ & {$\left[\begin{array}{lll}0.05 & 0\end{array}\right]^{T}[\mathrm{~m} / \mathrm{s}]$} \\
\hline Reference relative deviation $r_{r 1}$ & {$\left[\begin{array}{lll}0.3 & 0.3\end{array}\right]^{T}[\mathrm{~m}]$} \\
\hline Reference relative deviation $r_{r 2}$ & {$\left[\begin{array}{lll}0.3 & 0\end{array}\right]^{T}[\mathrm{~m}]$} \\
\hline Reference relative deviation $r_{r 3}$ & {$\left[\begin{array}{ll}0 & 0.3\end{array}\right]^{T}[\mathrm{~m}]$} \\
\hline Reference relative deviation $r_{r 4}$ & {$\left[\begin{array}{ll}0 & 0\end{array}\right]^{T}[\mathrm{~m}]$} \\
\hline Design parameter $k_{v r}$ & 0.23 \\
\hline Design parameter $\left[h_{1} h_{2}\right]$ & {$\left[\begin{array}{lll}2.0 & 2.0\end{array}\right]$} \\
\hline Controller gain $K$ & {$\left[\begin{array}{lllll}0.0012 & 0.63 & 0.011 & -0.012\end{array} \otimes I_{2}\right.$} \\
\hline Observer gain $H$ & {$\left[\begin{array}{lllll}3.25 & 0.96 & 1.51 & 1.10\end{array}\right]^{T} \otimes I_{2}$} \\
\hline
\end{tabular}

consensus error $L \hat{\tilde{r}}(t-\tau)$ and gives VV toward consensus because $L \hat{\tilde{r}}(t-\tau)$ converge equilibrium. And also Fig. 11 shows calculated velocities and gives that VVs achieve reference velocity.

\section{CONCLUSIONS AND FUTURE WORKS}

In this paper, a new control law of observer-based MAS is proposed for consensus problem. An agent has MIMO system, stability problem of MAS is extended from undirected graph to digraph. And also condition of stability was derived using lyapunov stability theorem. We consider MAS with communication delay that present a problem in MAS. Consensus problem is extended from state feedback to output feedback case. The proposed algorithm was applied to Multi-vehicle formation control problem to demonstrate the effectiveness of our proposed strategies.

\section{REFERENCES}

[1] Wei Ren, Randal W. Beard and Ella M. Atkins, "A Survey of Consensus Problems in Multi-agent Coordination," in Proc. ACC, pp.1859-1864, June. 2005.

[2] Richard Murray, "Recent Research in Cooperative Control of Multi-Vehicle Systems," Journal of Dynamic Systems, Measurement, and Control Vol. 129, Issue 5, pp.571-583. Sep. 2007.

[3] Wei Ren, "Consensus Based Formation Control Strategies for Multi-vehicle System," in Proc. ACC, pp.4237-4242, June. 2006.

[4] Roy S. Smith, Fred Y.Hadaegh “Closed-Loop Dynamics of Cooperative Vehicle Formations With Parallel Estimators and Communication," in Trans. AC, Vol.52, No. 8, pp. 1404-1414. Aug. 2007.

[5] Ather Gattami, Richard Murray, "A Frequency Domain Condition for Stability of Interconnected MIMO systems," in Proc. of American Control Conference, Boston, Massachusetts June 2004.

[6] Dongjun Lee and Mark W. Spong, "Stable Flocking of Multiple Inertial Agents on Balanced Graphs," in Trans. AC, Vol.52, No.8, pp. 1469-1475. Aug. 2007.

[7] Reza Olfati-Saber, J.Alex Fax, Richard Murray, "Consensus and Cooperation in Networked MultiAgent Systems," in Proc. IEEE, Vol. 95, No.1 pp.215-233. Jun, 2007.

[8] Reza Olfati-Saber and Richard Murray, "Consen-

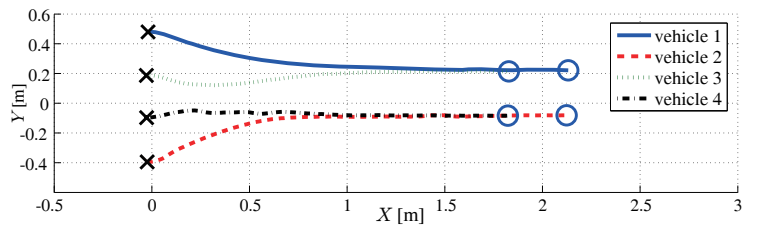

Fig. 6 Trajectories of four vehicles (Undirected graph)

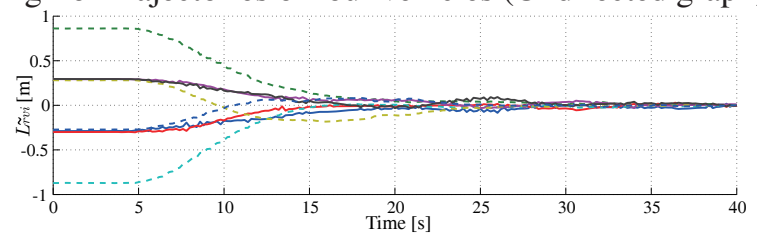

Fig. 7 Consensus error $L \tilde{r}_{v i}$ (Undirected graph)

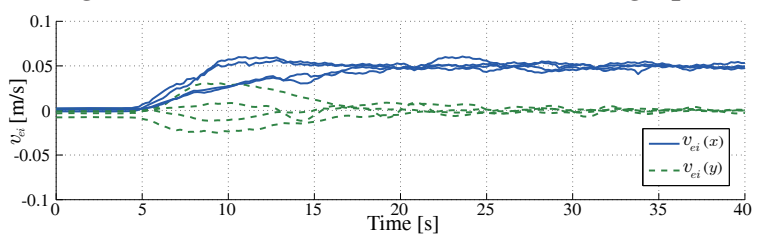

Fig. 8 Velocities of VVs $v_{e i}$ (Undirected graph)

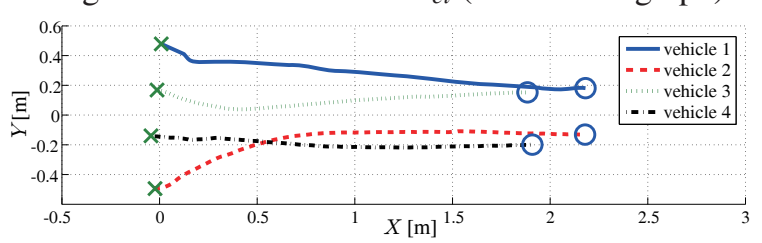

Fig. 9 Trajectories of four vehicles (Digraph)

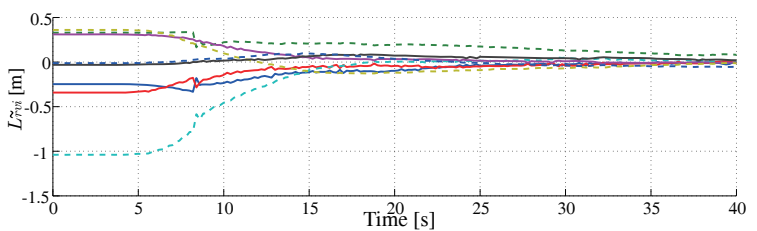

Fig. 10 Consensus error $L \tilde{r}_{v}(t-\tau)$ (Digraph)

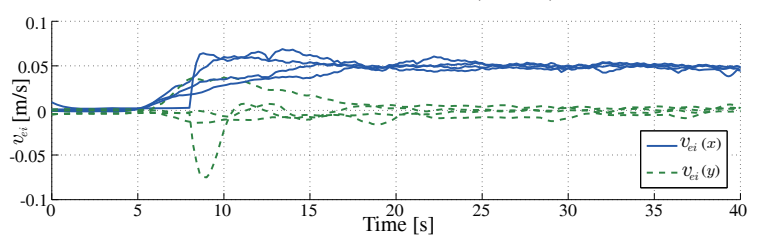

Fig. 11 Velocities of VVs $v_{e i}$ (Digraph)

sus Problems in Networks of Agents With Switching Topology and Time-Delays," IEEE Trans. AC, Vol. 49, No. 9, pp.1520-1532, 2004.

[9] Masateru Amano, Zhi-wei Luo, Shigeyuki Hosoe, "Graph-Dependent Sufficient Conditions for Synchronization of Network Coupled System with Time-delay," Trans. SICE, Vol. 40, No.9, pp. 925933, 2004.

[10] Rodolphe Sepulchre, Derek Paly and Naomi Leonard, "Collective Motion and Oscillator Synchronization," Cooperative Control, LNCIS 309, Vol. 309, pp.189-205, 2005.

[11] C. Godsil and G. Royle. Algebraic Graph Theory, volume 207 of Graduate Texts in Mathematics. Springer, 2001. 